\title{
When Participant Smoked, How many Cigarettes Smoked
}

National Cancer Institute

\section{Source}

National Cancer Institute. When Participant Smoked, How many Cigarettes Smoked. NCI

Thesaurus. Code C156833.

How many cigarettes did an individual smoke when they smoked. 